\title{
Finite element analysis of large rocket launcher
}

\author{
Deng Biao ${ }^{1}$, Guo Yuan ${ }^{1, a}$, Zhang $A n^{1}$,Tang Sheng-jin ${ }^{1}$ \\ ${ }^{1}$ Xi'an Institute of advanced technology, Xi'an 710025, China \\ aorchard1993@126.com
}

Key words: Rocket launcher structure; Finite element analysis; ANSYS

\begin{abstract}
By taking a certain type of rocket launcher structure as the research object, its loading analysis was carried out. By applying the ANSYS as a tool and by combining a typical working condition to carry out finite element analysis (FEA), the deformation and the stress values under the work condition were obtained, and the rocket launcher's strength and rigidity were analyzed. The results show that the research is based on the application of finite element software ANSYS calculation method on the launch pad is feasible, cost savings at the same time, the calculation result of finite element analysis can provide a theoretical basis for the subsequent upgrading of equipment.
\end{abstract}

\section{Introduction}

The rocket launcher is an important part of the whole transmission equipment, used to direct bearing rocket with verticality adjusting rocket verticality adjusting device, and the direction of machine driven rotary department to realize in the horizontal plane rotation, with azimuth aiming equipment of rocket launcher. Whether the design is reasonable, directly affect the bearing ability and performance of the launch pad. It is important to analysis and research on the mechanical structure of the launch pad.

The transmitter unit is shown in Figure 1. The transmitting station is mainly composed of frame, ring, raceway, steel ball, arm and jack support.The frame and bearing ring using standard steel and welded steel plate, arranged with a small amount of ribs to strengthen the stiffness, belonging to the welding parts, raceways are approximately rectangular billet rings. The bearing ring is welded on the frame and is fixedly connected with the frame, and the raceway is installed on the bearing ring by a bolt connection, and finally and finally the ball is assembled into one.It's center diameter is about $4250 \mathrm{~mm}$.
The traditional calculation method of the launch pad is based on the experience formula of the manual calculation[1][2], but because of the physical model of manual model is too simplified, the mathematical model of the mechanical characteristics of the structure does not fully comply with, so it can not guarantee the accuracy of the calculation. The finite element technology is very mature today,with the finite element software to calculate large complex structure, can greatly reduce the amount of labor and improve the accuracy of the calculation[3].At present, the finite element software is more and more widely used in the strong rigidity analysis of engineering machinery,but the analysis of the launch station is rare.In this paper, ANSYS finite element analysis software is used to calculate the static strength of the transmitting station, and a comprehensive understanding of the stress and deformation of the transmitter under full load is obtained. 


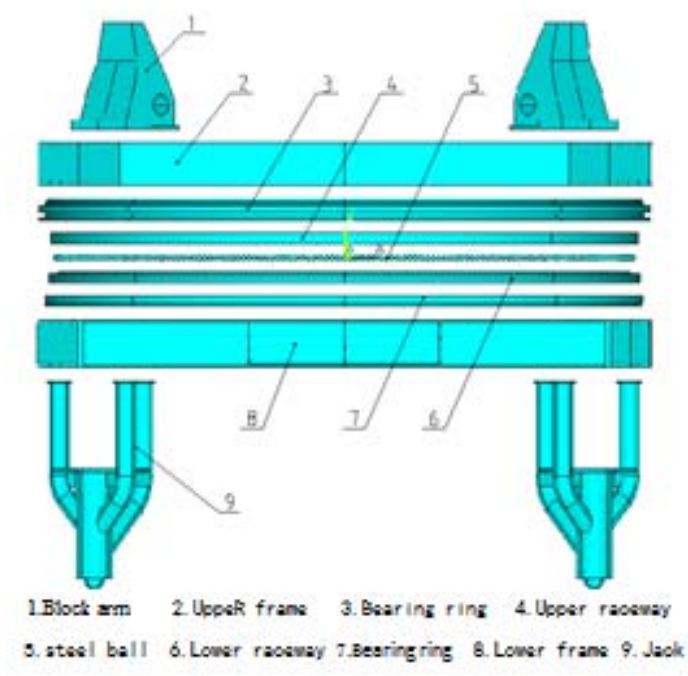

Fig. 1 Transmitter component

\section{The establishment of finite element model of transmitter}

\section{1 Structural simplification}

The accuracy and reliability of the finite element model have a direct impact on the quality of the mesh, which affects the accuracy of the results.Therefore, it is necessary to simplify the finite element analysis model of launching pad.Do the following simplification of the launch pad:

1)Ignoring the influence of steel plate welding stress and weld form.

2)Regardless of equipment installation error.

3)The through hole of the bearing rings to install the raceway has little influence on the overall stiffness and strength of the $\mathrm{C}$, so it is not considered。

Based on the above simplification, the finite element model of the transmitter is established in the finite element analysis software ANSYS(Fig.2).

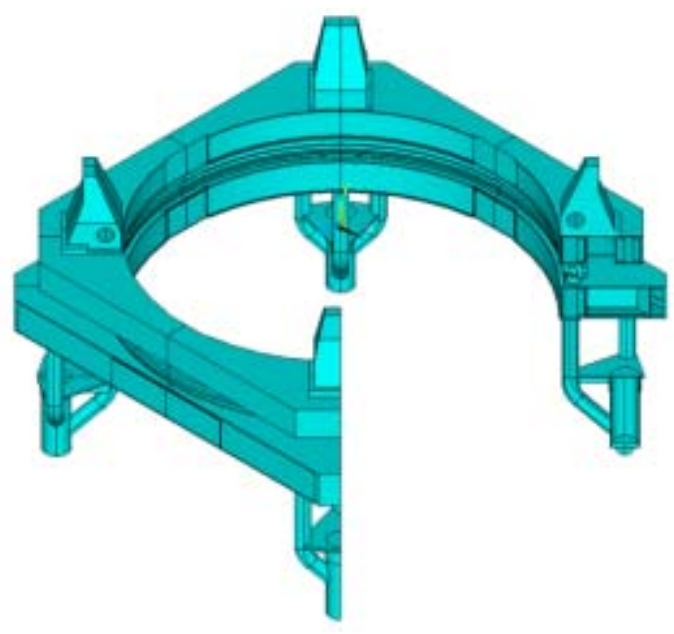

Fig. 2 Transmitter finite element model

\section{2 Unit type selection and meshing}

The finite element analysis method based on the software of the launching pad is to consider the structure of the launching pad as a linear model, and then give the finite element calculation of the strength and stiffness of the beam element,but the beam element is used to replace the three-dimensional solid structure [4], it does not reflect the geometric details of the structure, and the frame is a box-type structure made of welded steel and steel plates, Should be selected two-dimensional shell and shell structure and three-dimensional solid unit mixed network, or all selected three-dimensional solid element division grid.Considering the complexity of the transmitter model, the finite element analysis of the transmitting station is carried out by using the three-dimensional solid element Solid185.From the overall structure of the launcher, the upper and lower frame is mainly through the ball and raceway contact and extrusion to transmit force, Therefore, it is necessary to solve the contact problem between the ball and the raceway. The 3 point contact element Conta178 element is used to simulate the steel ball.

The Solid185 element is a high order three-dimensional 8 node solid element, and 
each node has a 3 degree of freedom.It has the function of dealing with physical phenomena such as plasticity, super elasticity, stress hardening, creep, large deformation and large strain [5]. When the structure of the irregular boundary shape is divided, the unit can be degenerated into a prism shape, a tetrahedral shape, a Pyramid shape, and so on, that is, the 8 nodes in the original node can overlap. The unit requires simple input data, can be applied to the surface pressure load, the output data is comprehensive, to meet our structural node deformation and stress calculation requirements, but also one of the most widely used units[6].

The conta178 contact element can be used to deal with the contact and slip of two types of elements, and each node of the element has 3 degrees of freedom, which supports the normal extrusion and tangential Coulomb friction between the contact surfaces [7].In this structure, it can not only satisfy the transmission of the upper and lower frame, but also give the pressure of the single steel ball.Considering the symmetry of the whole structure and the difference of the radius of curvature of the raceway and the radius of the steel ball, it is reasonable and effective to use the unit to simulate.Conta178 unit provides enhanced
Lagrange method, penalty function method, pure Lagrange multiplier method and other 4 kinds of contact algorithm.In this paper, we use the enhanced Lagrange method, which is given in the element attribute selection[8].

The thickness of the mesh will seriously affect the analysis results, the finer the mesh, the more accurate the results will be, but the calculation time will increase accordingly.In this paper, using the technology of Free, the main part of the launching pad is divided into 236775 nodes, and the number of units is 869372 .

\section{3 Material definition}

The Launch pad structural material parameters in Table 1.

Table 1 Structure parameters of launching pad

\begin{tabular}{ccccc}
\hline Member & Material & $\begin{array}{c}\text { Elastic } \\
\text { modulus } / \mathrm{Pa}\end{array}$ & Poisson ratio & Density $/\left(\mathrm{Kg} / \mathrm{m}^{3}\right)$ \\
\hline Frame & $16 \mathrm{Mn}$ & $2.12 \times 10^{11}$ & 0.31 & 7870 \\
Ring & $35 \#$ & $2.12 \times 10^{11}$ & 0.29 & 7870 \\
Racewa & $5 \mathrm{CrMnM}$ & $2.07 \times 10^{11}$ & 0.25 & 7870 \\
$\mathrm{y}$ & $\mathrm{o}$ & & & \\
\hline
\end{tabular}




\section{4 Load and constraint processing}

The load acting on the launching pad is divided into conventional load, accidental load, special load and other loads. Since this is a static strength analysis of the launch pad, the load required for the calculation of the transmitter is only the weight load in the conventional load.As the rocket placed high verticality,so that the load calculation is the vertical load (axial load). The total weight of the rocket is $\mathrm{Q}$, according to the structural characteristics of the launch pad, the load is decomposed into 4 evenly distributed arms on the upper frame, and the force of a single arm is shown in Figure 3.Parameters $R_{1}, R_{2}$, $\mathrm{h}$ are known, by the force, torque balance available:

$$
N=\frac{Q}{4} \quad F=\frac{Q\left(R_{1}-R_{2}\right)}{4 \mathrm{~h}}
$$

In type:

$$
\mathrm{Q} \text { - } \text { rocket gravity, } N \text {; }
$$

$\mathrm{N}-$ - vertical support reaction of $\operatorname{pin}, N$;

$\mathrm{F}$-—arm horizontal reaction, $\mathrm{N}$;

$\mathrm{R}_{1}$ - - horizontal distance from pin to center, $\mathrm{mm}$;

$\mathrm{R}_{2}$ - - horizontal distance from the support point to the center of rotation, $\mathrm{mm}$;

$\mathrm{h}-$ - the vertical distance from the horizontal arm to the center of the pin axis, $m m$

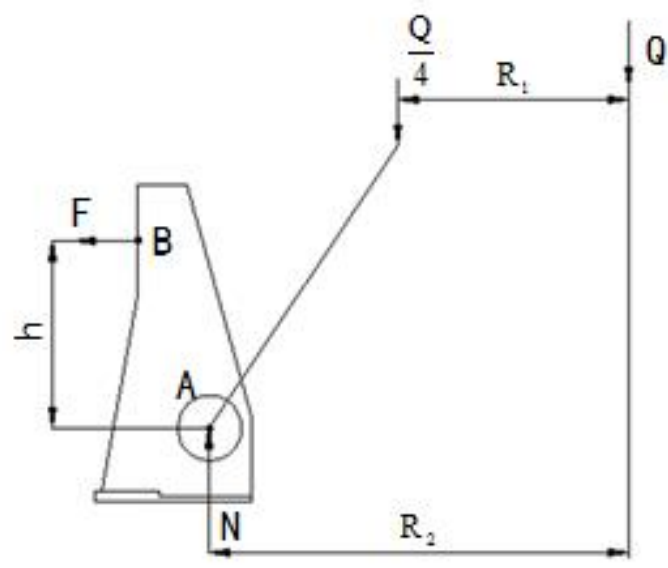

Fig. 3 Block arm force diagram

In the case of full load, it is the most destructive to the structure of the launching pad when the arm and the jack support are distributed 450.Therefore, the working condition is determined.

Because the jack mounting base is a ball hinge support,the two parts are only allowed to rotate relative to the public sphere, limit their relative movement in three directions. The load acting on the launching pad can be applied to the retaining arm according to the calculated values and the corresponding conditions.For the gravity of the launch pad,ANSYS software will be calculated on the basis of density and gravity acceleration,without additional application.

\section{Calculation and analysis of the results}

According to the structure, load constraints and other actual situation,the results are given in reference to the cylindrical coordinate system, its origin is the same as that of the original rectangular coordinate system. The $\mathrm{X}$ axis is radial, the $\mathrm{Z}$ axis is upward along the central axis, and the $\mathrm{Y}$ axis represents the tangential direction of the circle.

It can be seen by calculating the results of the analysis, the maximum equivalent stress is $669.8 \mathrm{MPa}$ when the arm and the jack support are distributed 450.The equivalent stress distribution of the launching pad is shown in Figure 4.The maximum equivalent stress appears in the 
jack ball hinge support(as shown at $E$ in Figure 5).

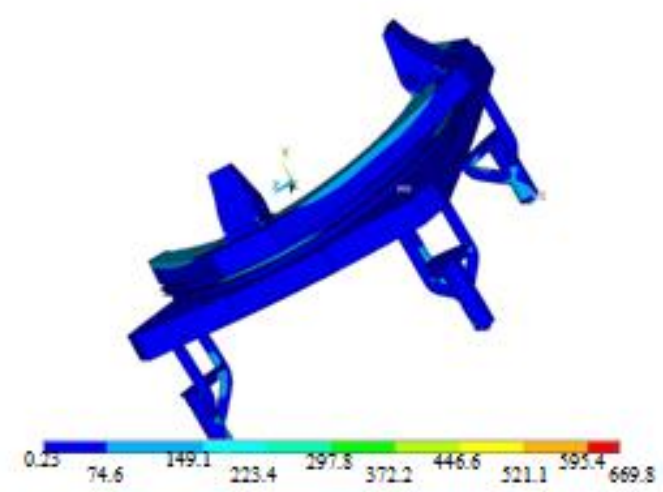

Fig. 4 Equivalent stress of transmitting station

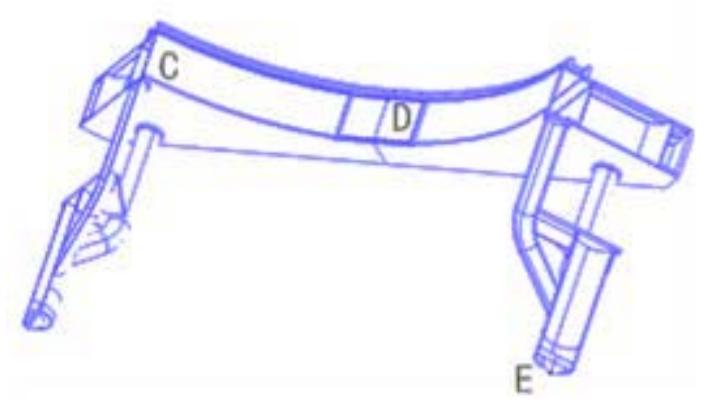

Fig. 5 Lower frame partial model

The equivalent stress distribution and deformation of the upper frame are shown in Figure 6.The maximum equivalent stress is 288.9Mpa, which appears on the inner side of the arm(as shown at A in Figure 7). The maximum deformation is $2.63 \mathrm{~mm}$ (as shown at $B$ in Figure 7).

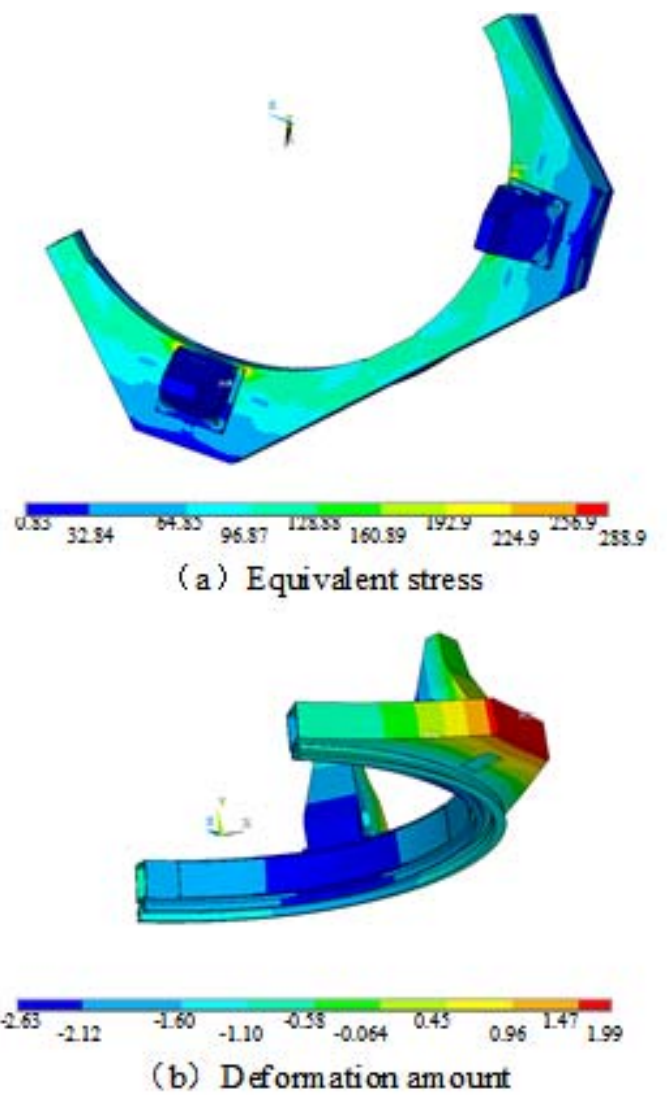

Fig. 6 Equivalent stress and deformation of upper frame

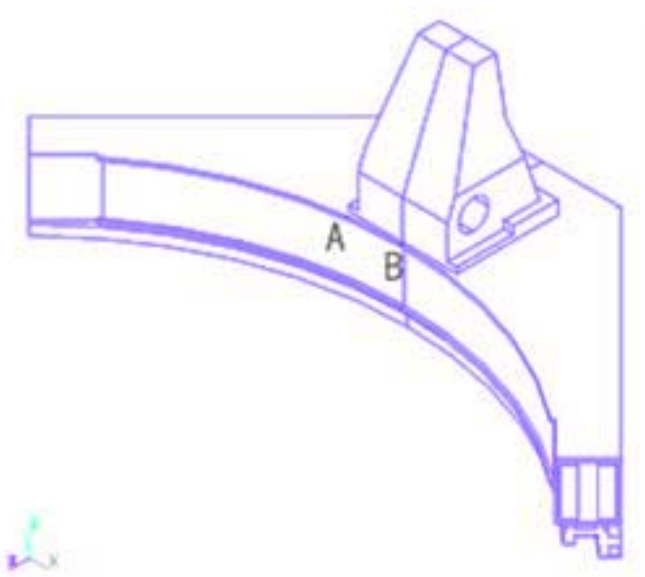

Fig. 7 Upper frame model

The equivalent stress distribution and deformation of the lower frame are shown in Figure 8.The maximum equivalent stress is $115 \mathrm{Mpa}$, which appears at the top of the jack(as shown at $\mathrm{C}$ in Figure 5).The maximum deformation is $1.75 \mathrm{~mm}$ (as shown at $\mathrm{D}$ in Figure 5). 


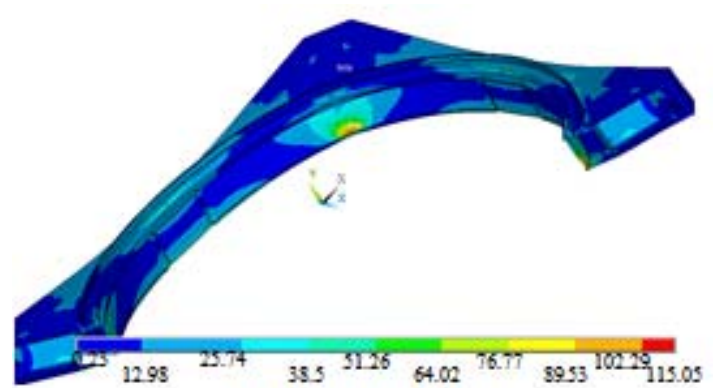

(a) Equivalent stress

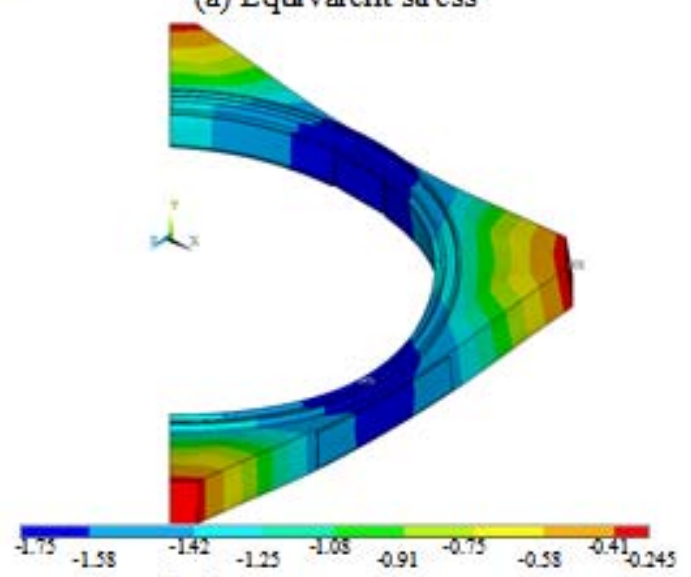

(b) Deformation amount

Fig. 8 Equivalent stress and deformation of lower frame

The equivalent stress distribution and deformation of the raceway are shown in Figure 9.The maximum equivalent stress is $1373 \mathrm{Mpa}$, which appears on the outside of the raceway(as shown at $\mathrm{F}$ in Figure 10). The maximum deformation is $2.03 \mathrm{~mm}$, which appears at the bottom of the arm (as shown at $\mathrm{G}$ in Figure 10).
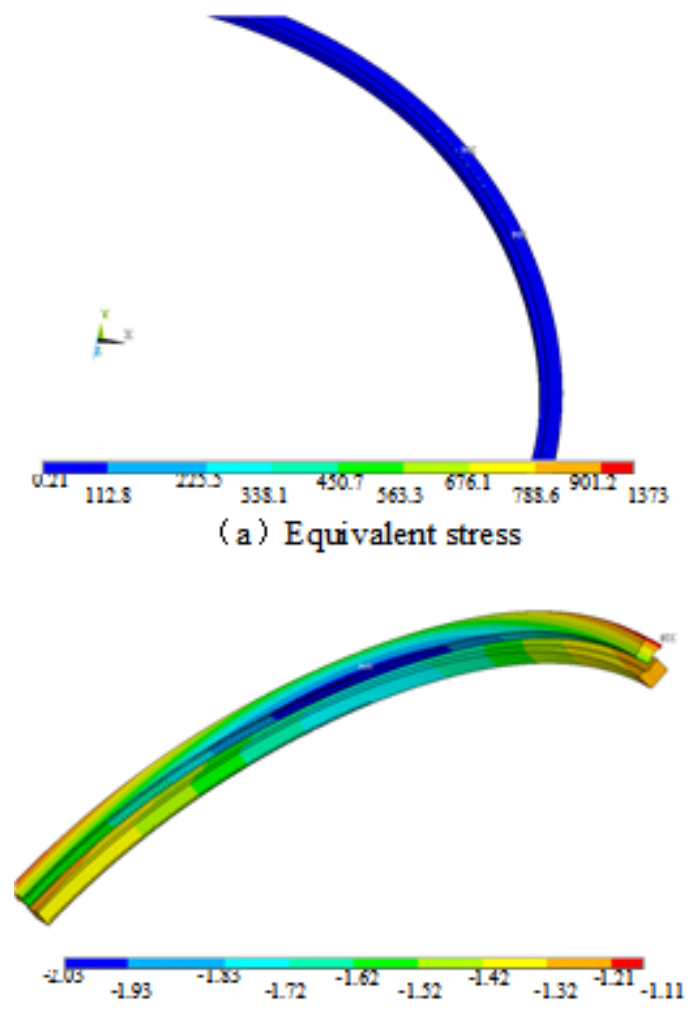

(b)D eformation amount

Fig. 9 Equivalent stress and deformation of raceway

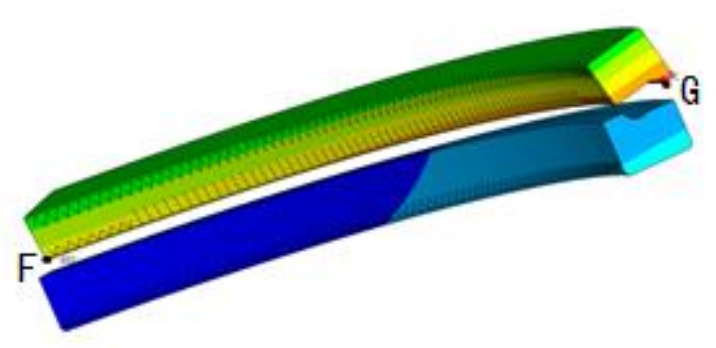

Fig. 10 Raceway model

The maximum equivalent stress and the maximum deformation of each component are shown in Table 2.

Table 2 Maximum equivalent stress and deformation of each component

\begin{tabular}{ccc}
\hline Member & Maximum equivalent stress $/ \mathrm{MPa}$ & Maximum deformation $/ \mathrm{mm}$ \\
\hline Upper frame & 288.9 & 2.63 \\
Upper raceway & 1373 & 2.03 \\
Lower frame & 115 & 1.75 \\
Lower raceway & 1014 & 1.72 \\
\hline
\end{tabular}




\section{1 Strength analysis}

The frame material is $16 \mathrm{Mn}$,yield limit $\sigma_{\mathrm{s}}=343 \mathrm{Mpa}$ allowable stress $[\sigma]=\sigma_{\mathrm{s}} / 1.5=229$ Mpa.As can be seen from the stress clouds in Figures 6 and 8, the maximum equivalent stress of the upper and lower frames is 288.9 Mpa,115 Mpa.Both are less than the yield limit $\sigma_{\mathrm{s}}$, but the latter is greater than the allowable stress $[\sigma]$.Taking into account the role of the arm in the horizontal reaction, $\mathrm{A}$ (in figure 7) is mainly subjected to compressive stress. The compressive strength of brittle material is generally greater than the tensile strength, the compressive strength limit is $4 \sim 5$ times of tensile strength.Therefore, the framework still meets the strength requirements. The maximum equivalent stress of the launcher is $669.8 \mathrm{Mpa}$,which appears in the jack ball hinge support(as shown at $\mathrm{E}$ in Figure 5).In the actual situation, the mounting base of the jack is a ball hinge support,which is mainly subjected to compressive stress, and the stress of the base plate is greatly lower in the welding process. So the position meets the strength requirements. The raceway material is $5 \mathrm{CrMnMo}$,yield limit $\sigma_{\mathrm{s}}=1445 \mathrm{Mpa}$,allowable stress $[\sigma]=\sigma_{\mathrm{s}} / 1.5=963 \mathrm{Mpa}$. The maximum equivalent stress of raceway is $1373 \mathrm{Mpa}$,which is less than the yield stress and greater than allowable stress. The upper and lower raceway through the ball contact transmission force and movement.The contact stress between steel ball and raceway is compressive stress.So the raceway meets the strength requirements.

In summary, the launch pad components meet the strength requirements.

\section{2 Stiffness analysis}

From the above calculation results we can see,in the case of full load, the axial displacement of the upper frame is larger $(2.63 \mathrm{~mm})$, and the stiffness is weak. The loading mode of the launching pad makes the upper frame, upper bearing ring and the upper raceway bear the bending and torsion combination. The upper and the lower roller tracks have a serious radial displacement, relative to the center of rotation, the load bearing arm is internally twisted, so that the upper roller track is turned outwards to move, and the lower roller path moves inwards.As shown in figure 11, the maximum radial misalignment of the upper and lower raceways is $0.92 \mathrm{~mm}$. The dislocation of the upper and lower raceway will directly affect the movement of the steel ball in the raceway.In severe cases, the ball can be stuck in the raceway, making the launch pad difficult to slip or even stuck.In addition, the loading condition of the launcher is special. If the load is in full load condition for a long time, the elastic deformation will accumulate to the plastic deformation which can not be recovered,seriously affecting the normal work of the launch pad.

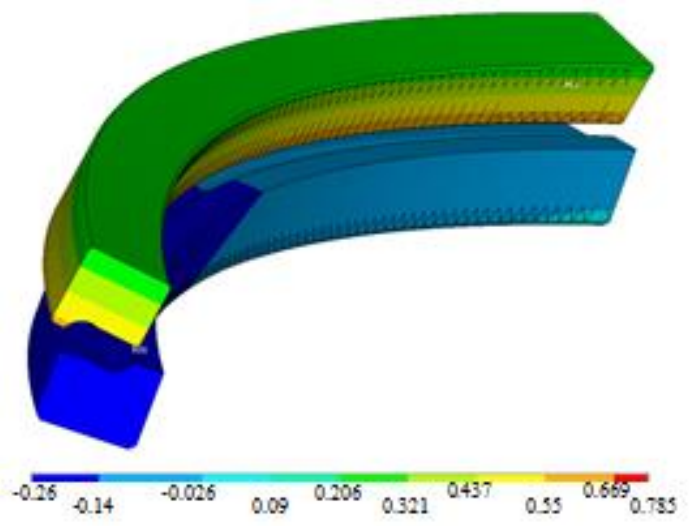

Fig. 11 Local deformation of raceway

\section{Summary}

In this paper, the structure of the rocket launcher is modeled by using the general finite element analysis software ANSYS.The strength and stiffness of the launching pad are analyzed according to the actual load characteristics. The main reason for the abnormal rotation is analyzed theoretically.In addition, based on the 
analysis of the stress and strain distribution,the structure of the launcher can be optimized by changing the thickness of the plate, the cross section shape, and the replacement of the new slewing bearing,this provides a very valuable reference to the subsequent transformation and upgrading.

\section{References}

[1] Shao Shixiang. A simple calculation method for multi support launching platform [j]. missile and space launch technology, 1998 (1): 28-34.

[2] Shao Shixiang. A simple method for calculating the strength and stiffness of $\mathrm{CZ}$ - 2E launcher [J]. missile and space launch technology, 1994 (1): 37-44.

[3] Shang Zhenguo, Dong Huimin, et al., et al. Finite element analysis method of turntable bearing with plastic deformation [J]. Chinese Journal of agricultural engineering, 2011, 27 (): 52-56.

[4] Xiong Ying, Chen Deyong. Finite element analysis on the strength of the pedestal and the failure of the slewing bearing in the ladle $[\mathrm{J}]$. heavy machinery, 2015 (2): 75-78.

[5] Haining, Wang Gang. Finite element analysis of MW class wind turbine gearbox $[\mathrm{J}]$. mechanical transmission, 2012, 36 (4): 70-72.

[6] Xu Bin, Chen Nan, Wang Hui, et al. Finite element analysis of complex mechanism based on contact element method $[\mathrm{J}]$. Journal of Southeast University, Natural Science Edition, 2009, 39 (3): 495-501.

[7] Yao Keheng, Yin Chenbo. On the platform of ANSYS tower crane jib in finite element analysis and Research on the key technology of [J]. modern manufacturing engineering, 2009 (12): 47-51.

[8] Wei Shifu, Wang Sanmin,, et al. Finite element analysis and experimental verification of a crane boom [J]. mechanical design, 2011, 28 (6): 92-96. 